\title{
Caracterizacion, simulacion y propuesta de mejora del proceso de reinscripcion en el Instituto Tecnologico Superior de Huatusco
}

\section{Simulation and improvement proposal for Instituto Tecnologico Superior de Huatusco reinscription process}

\author{
SOLIS-JIMENEZ, Miguel Ángel†*, CALDERÓN-PALOMARES, Luis Antonio, LOPEZ-LEON, Alí y
} GONZÁLEZ-SOBAL, Martín

Instituto Tecnológico Superior de Huatusco / Tecnológico Nacional de México. Av. 25 Poniente No. 100 Colonia Reserva Territorial, Huatusco. Ver. CP. 94100

ID 1 ${ }^{\text {er }}$ Autor: Miguel Ángel, Solís-Jimenez / ORC ID: 0000-0002-8125-0989, CVU CONACYT ID: 94216

ID $1^{\text {er }}$ Coautor: Luis Antonio, Calderón-Palomares / ORC ID: 0000-0001-9846-5567, Researcher ID Thomson: N-62592018 ,

CVU CONACYT ID: 238274

ID $2^{\text {do }}$ Coautor: Ali, López León / CVU CONACYT ID: 661438

ID $3^{\text {er }}$ Coautor: Martín, Gonzalez-Sobal / ORC ID: 0000-0003-0038-8319, Researcher ID Thomson: N-6243-2018, CVU CONACYT ID: 463431

DOI: $10.35429 / J U M .2019 .7 .3 .22 .31$

Recibido 05 de Enero, 2019; Aceptado 30 de Marzo, 2019

\begin{abstract}
Resumen
Uno de los procesos claves, hablando de servicio a los alumnos en las instituciones de educación, es el proceso de reinscripción. En el Tecnológico Superior de Huatusco esta acción aún se lleva a cabo con la participación in situ de los alumnos, donde ellos pasan por diversos procesos hasta quedar legalmente inscritos para el semestre siguiente. Una actividad crítica en el proceso tiene que ver con la asesoría reticular, donde los alumnos pasan con los docentes y tomando en cuenta el avance reticular, así como las materias ofertadas, se le asigna al alumno la cantidad de créditos que más les convenga, buscando con esto lograr un avance constante en su carrera. Los alumnos se clasifican en regulares e irregulares, y de acuerdo a esta clasificación es el tiempo que se requiere para llevar a cabo la asesoría reticular, ya que un alumno regular consume menor cantidad de tiempo que un alumno irregular. Se han efectuado diversos esfuerzos para agilizar la asesoría reticular, pero los resultados no han sido muy alentadores. Utilizado un modelo de simulación se busca encontrar la reingeniería adecuada que permita reducir el tiempo de asesoría y con ello reducir las quejas de los alumnos en este proceso.
\end{abstract}

Simulación, Caracterización, Reingeniería

\begin{abstract}
One of the key processes, about service to students in educational institutions, is the re-enrollment process. At the Tecnologico Superior de Huatusco, this action is still carried out with the participation in situ of the students, where they go through various processes until they remain legally registered for the following semester. A critical activity in the process has to do with the reticular counseling, where the students spend with the teachers and taking into account the reticular progress, as well as the subjects offered, the student is assigned the number of credits that suits them, looking for With this achieve a steady advance in your career. Students are classified as regular and irregular, and according to this classification is the time required to carry out the reticular counseling, since a regular student consumes less time than an irregular student. Various efforts have been made to expedite reticular counseling, but the results have not been very encouraging. Using a simulation model, we seek to find reengineering that allows us to reduce counseling time and thereby reduce the complaints of students in this process.
\end{abstract}

Simulation, Characterization, Reengineering

Citación: SOLIS-JIMENEZ, Miguel Ángel, CALDERÓN-PALOMARES, Luis Antonio, LOPEZ-LEON, Alí y GONZÁLEZ-SOBAL, Martín. Caracterizacion, simulacion y propuesta de mejora del proceso de reinscripcion en el Instituto Tecnologico Superior de Huatusco. Revista de Gestión Universitaria. 2019. 3-7: 22-31

\footnotetext{
* Correspondencia del Autor (correo electrónico: miguel_solis@itshuatusco.edu.mx)

$\dagger$ Investigador contribuyendo como primer autor.
} 


\section{Introducción}

La simulación es una forma de estudiar los procesos aleatorios, los cuales se encuentran prácticamente en todas las operaciones del sistema de producción y servicios. Sin duda, la flexibilidad en cuanto a la modelación, el análisis de los datos y el mejoramiento del sistema han hecho de la simulación una herramienta cuyo uso y desarrollo se han visto alentadas de manera significativa.

El concepto simulación denota las acciones de imitar, copiar o representar cualquier cosa. Básicamente, ésta es la esencia de las siguientes definiciones generadas por diversos especialistas en el área de simulación con un enfoque práctico.

Desde un punto de vista amplio, Thomas H. Naylor la define así:

Simulación es una técnica numérica para conducir experimentos en una computadora digital. Estos experimentos comprenden ciertos tipos de relaciones matemáticas y lógicas, las cuales son necesarias para describir el comportamiento y la estructura de sistemas complejos del mundo real a través de largos periodos de tiempo. (Coss Bu, 1990)

En un sentido más estricto y con un enfoque sistémico, Robert E. Shannon, la define como:

Simulación es el proceso de diseñar y desarrollar un modelo computarizado de un sistema o proceso y conducir experimentos con este modelo con el propósito de entender el comportamiento del sistema y evaluar varias estrategias con las cuales se puede operar el sistema. (Coss $\mathrm{Bu}, 1990)$

Existen muchos otros conceptos acerca de la simulación y todos coinciden en que la simulación hace uso de una computadora para estudiar un sistema numéricamente mediante un modelo en un periodo de tiempo deseado.

\section{Sistemas y modelos}

Un sistema es un conjunto de entidades que interactúan entre sí para cumplir con un propósito en común. Cualquier sistema puede ser dividido en subsistemas con características y variables suficientes para realizar un estudio, llamándose a este último el sistema bajo estudio.
Las limitaciones que definen a un sistema están basadas en el objetivo de estudio.

Los sistemas pueden ser clasificados en dos tipos: discretos y continuos. Un sistema discreto es aquél en el cual las variables cambian en puntos separados a través del tiempo y un sistema continuo, en el cual las variables cambian continuamente en el tiempo.

Independientemente de esta clasificación, existe una generalidad de los diferentes caminos bajo los cuales pueden ser estudiados.

De acuerdo a Carillo (1997), un modelo es la interpretación explícita de lo que se entiende de una situación. Puede expresarse mediante ecuaciones matemáticas, símbolos o palabras, pero en esencia es una descripción de entidades, procesos o atributos y las relaciones entre ellos. Puede ser descriptivo o ilustrativo, pero sobretodo debe ser útil para cumplir los objetivos de estudio planteados.

Azarag y García (1996) establecieron la siguiente clasificación de modelos:

- Modelos icónicos. Son los modelos físicos que se asemejan al sistema real, generalmente manejados a escala.

- Modelos análogos. Son los modelos en los que una propiedad del sistema real se puede sustituir por una propiedad diferente que se comporta de manera similar.

- Modelos simbólicos. Son aquellos en los que se utiliza un conjunto de símbolos en lugar de una entidad física para representar a la realidad. Dentro de estos modelos, se encuentran los modelos utilizados en simulación y a su vez se clasifican en:

- Modelos determinísticos. En estos modelos, los valores de las variables no se ven afectados por variaciones aleatorias y se conocen con exactitud.

Modelos estocásticos. Los valores de las variables dentro de un modelo estocástico sufren modificaciones aleatorias con respecto a un valor promedio; dichas variaciones pueden ser manejadas mediante distribuciones de probabilidad.

SOLIS-JIMENEZ, Miguel Ángel, CALDERÓN-PALOMARES, Luis Antonio, LOPEZ-LEON, Alí y GONZÁLEZ-SOBAL, Martín. Caracterizacion, simulacion y propuesta de mejora del proceso de reinscripcion en el Instituto Tecnologico Superior de Huatusco. Revista de Gestión Universitaria. 2019 
- Modelos dinámicos. La característica de estos modelos es el cambio que representan las variables en función del tiempo; por ejemplo los modelos de series de tiempo y pronósticos.

- Modelos estáticos. En este tipo de modelos no se maneja la variable tiempo, esto es, representan a un sistema en un punto particular del tiempo.

Modelos continuos. Son modelos en los que las variables pueden tomar valores reales y manejarse mediante las técnicas de optimización clásica.

- $\quad$ Modelos discretos. Aquí las variables del sistema toman valores sólo en el rango de números enteros.

\section{Etapas para realizar un estudio de simulación}

En la metodología para la creación y desarrollo de un modelo de simulación, Pritsker y Pedgen (1979) resaltan la naturaleza de este proceso en las etapas de:

$\begin{array}{ll}\text { 1. } & \text { Plan de estudio } \\ \text { 2. } & \text { Definición del sistema } \\ \text { 3. } & \text { Construcción del modelo } \\ \text { 4. } & \text { Diseño de experimentos } \\ \text { 5. } & \text { Análisis de los resultados } \\ \text { 6. } & \text { Reporte de resultados }\end{array}$

\section{Errores al realizar un estudio de simulación}

Asumiendo que la decisión de utilizar la técnica de simulación ha sido tomada, se debe tener especial cuidado al aplicarla en no cometer los siguientes errores más comunes descritos por Law (2000):

- Falla en la definición clara de los objetivos del estudio de simulación.

Nivel inapropiado de detalle del modelo. Falta de comunicación con los administradores durante el proceso de simulación.

- Mal entendimiento de la simulación por parte de los administradores.

Tratar a la simulación como un solo ejercicio de programación.

Falta de conocimientos de la metodología de simulación y conocimientos sobre estadística.

- Mala colección de datos del sistema.

- $\quad$ Software de simulación inadecuado.
- Mala documentación de las macros utilizadas durante la simulación lo que provocan la mala implementación.

- Creer que la simulación es una técnica demasiado compleja y desecharla por falta de conocimiento de ésta.

- Demasiada confianza en simuladores que hacen accesible la simulación a todo el mundo, de manera que se piense que cualquiera puede hacer un estudio de simulación.

- Correr una sola vez el programa, sin realizar un cuidadoso diseño de experimentos.

- Utilizar medidas de desempeño del sistema erróneas, que no reflejen la situación real del sistema que se estudia, por lo tanto, las decisiones que se tomen basadas en éstas medidas de desempeño no serán de utilidad ni conllevarán a mejorar el mismo.

- Mal uso de la animación, y por ende rechazo de la simulación por no hacerla seria. Se debe tratar en lo posible que la animación sea lo más realista, a fin de hacer más entendible el modelo del sistema bajo estudio, sin descuidar todo sustento estadístico de dicho modelo.

Actualmente la simulación presta un invalorable servicio en casi todas las áreas posibles y esto sin duda se debe su flexibilidad. La simulación es una excelente herramienta para ayudar a comprender un sistema real debido a que puede expandir, comprimir o detener el tiempo, y además es capaz de brindar información sobre variables que no pueden ser medidas o sería muy complejo medirlas en el sistema real. En el presente trabajo que lleva por nombre "Caracterización, simulación y propuesta de mejora del proceso de reinscripción de la carrera de Ingeniería Industrial en el Instituto Tecnológico Superior De Huatusco" pretende poner atención minuciosa a cada uno de los procesos que comprenden el proceso de reinscripción, para con ello tratar de entender cómo es que el sistema está operando y con esto dar una propuesta de solución a las problemáticas que se manifiestan en el sistema, tales como: inconformidad de los estudiantes y del personal encargado del servicio ya que es muy lento (en ocasiones el estudiante tarda hasta 2 horas para completar el proceso, donde se debe de pasar por una serie de actividades y hacer varias filas presentándose varios cuellos de botella). 
Por otra parte, existe una incorrecta distribución del trabajo entre las personas que participan del proceso, ya que mientras algunos tienen trabajo todo el tiempo, otros se encuentran desocupados ocasionando inconformidad entre las personas.

El modelo que se desarrolla muestra las áreas que intervienen en el proceso de reinscripción y se busca identificar en cual se presentan mayor número de inconsistencias. De igual forma, explica cómo se clasificaron a los alumnos de acuerdo al servicio que requiere cada uno; el proceso que debe seguir durante la reinscripción; así como se realizan los muestreos correspondientes y analizar cada una de las variables para determinar cuál distribución de probabilidad están siguiendo y utilizar esta información para obtener el modelo de simulación.

A partir del modelo verificado $\mathrm{y}$ validado, se hacen propuestas de mejora buscando reducir los tiempos de espera, equilibrar la carga de trabajo y, por ende, optimizar el proceso de reinscripción.

\section{Metodología a desarrollar}

El proceso que se siguió para el desarrollo del proyecto, consistió en primer lugar en identificar las partes en que se divide el proceso de reinscripción de manera general, el proceso se divide en:

Entrega de boletas.

Los alumnos llegan a formarse por semestre y carrera para que reciban su boleta de calificaciones, donde los recibirán 4 personas para agilizar el proceso.

\section{- $\quad$ Cotejo de pago.}

Después de que le proporcionen su boleta, el alumno deberá acudir a la sección de cotejo de pago el cual consiste en que el alumno mostrará a la persona correspondiente, que realizó el pago de reinscripción generado en la $\mathrm{OVH}$ (Hacienda) y el pago en el banco.

\section{- $\quad$ Asesoría reticular.}

En esta etapa es la más complicada, ya que los alumnos se dirigirán a un asesor para que les realice su carga académica de acuerdo al número de materias aprobadas; donde cuatro académicos serán quienes realicen la asesoría correspondiente.

\section{Autorización.}

Aquí se concluye el proceso con el sello y firma donde les están permitiendo la realización de la carga académica.

La Figura 1, muestra la distribución un poco más detallada del área de inscripción.

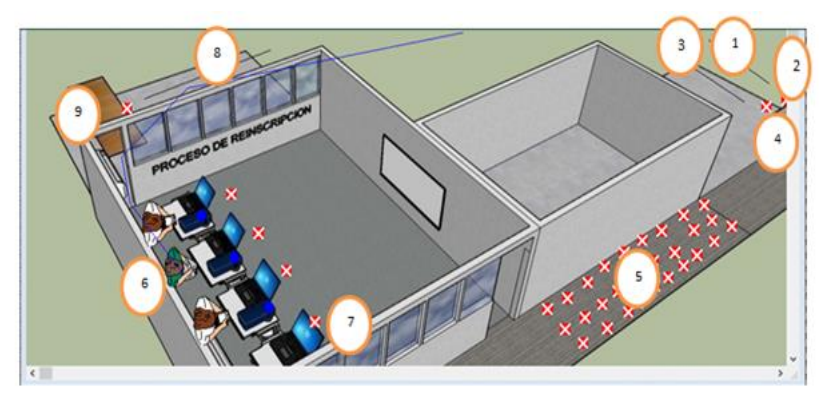

Figura 1 Distribución del área de reinscripción Fuente: Elaboración Propia

Las áreas marcadas son las siguientes:

- Llegada de los alumnos a entrega de boletas.

- $\quad$ Entrega de boletas,

- $\quad$ Fila de servicios financieros;

- $\quad$ Cotejo de pago.

- Zona de espera, antes de asesoría reticular.

- $\quad$ Servidores en donde estarán docentes asesorando a los alumnos para generar su carga académica,

- Fila de espera para que puedan ser atendidos los alumnos irregulares.

- $\quad$ Fila de espera de servicios escolares;

- Atención a servicios escolares para la autorización de la carga académica;

El proyecto se centra en el tercer proceso, es decir en la asesoría reticular. Ya que es este punto se genera el mayor tiempo de espera, dicho en otras palabras, es donde se forma el mayor cuello de botella. 
Los alumnos se clasifican para el estudio de la siguiente manera: de acuerdo al semestre que van a cursar, y si llevan materias reprobadas o no; si el alumno lleva una o más materias no acreditada serán alumnos irregulares, de igual forma serán considerados como irregulares a los alumnos convalidados. Por lo tanto, los alumnos que se tienen son:

\section{- $\quad$ Alumno tercer semestre regular. \\ - $\quad$ Alumno tercer semestre irregular. \\ - Alumno quinto semestre regular. \\ - Alumno quinto semestre irregular. \\ - Alumno séptimo semestre regular. \\ - Alumno séptimo semestre irregular.}

Esta clasificación es conveniente para comprender el sistema, ya que mientras más adelantado en semestres estén los alumnos, el tiempo que requiere un alumno irregular para su inscripción es mayor.

\section{Plan de muestreo y recolección de datos}

Para la recolección de datos en el proceso de reinscripciones se realizó en el sistema sabatino, ya que este sistema es donde mayor población estudiantil se tiene y por ende mayor cantidad de inconformidades. Cabe destacar que este proceso se lleva a cabo dos veces al año, al inicio de cada semestre. Se tomaron los datos de la muestra en el proceso de reinscripción del periodo febrero-julio 2018 y agosto-enero 2019.

Una vez que se tiene definido el proceso, así como las variables aleatorias, se procedió a la toma de tiempos de los servicios, así como la llegada de los alumnos a la primera fila. Con estos datos, se hicieron las pruebas de bondad de ajuste, para determinar el tipo de variable aleatorio que siguen los datos. Los resultados se presentan en las siguientes tablas:

\begin{tabular}{|l|r|}
\hline Media & 1.666 \\
\hline $\begin{array}{l}\text { Desviacion Estandar } \\
\text { Distribucioin }\end{array}$ & 5.736 \\
\hline
\end{tabular}

Tabla 1 Distribución de tiempo de entrega de boletas Fuente: Elaboración Propia

\begin{tabular}{|l|r|}
\hline Media & 0.454 \\
\cline { 2 - 2 } $\begin{array}{l}\text { Desviacion Estandar } \\
\text { Distribucioin }\end{array}$ & 0.208 \\
\hline
\end{tabular}

Tabla 2 Distribución de tiempo de cotejo de pago Fuente: Elaboración Propia

\begin{tabular}{|l|r|}
\hline Media & 0.528 \\
\hline $\begin{array}{l}\text { Desviacion Estandar } \\
\text { Distribucioin }\end{array}$ & 0.296 \\
\hline
\end{tabular}

Tabla 3 Distribución de tiempo de Autorización de carga Fuente: Elaboración Propia

Con relación a los tiempos de servicio dentro del proceso de asesoría reticular se tiene

\begin{tabular}{|l|l|}
\hline \multicolumn{2}{|c|}{ Regular Tercero } \\
\hline Media & 1.720 \\
\hline Desviacion Estandar & 1.113 \\
\hline Distribucioin & Lognormal \\
\hline Regular Quinto \\
\hline Media \\
\hline Desviacion Estandar & 3.287 \\
\hline Distribucioin & Lognormal \\
\hline Regular Septimo \\
\hline Media & 1.857 \\
\hline Desviacion Estandar & 1.069 \\
\hline Distribucioin & Lognormal \\
\hline
\end{tabular}

Tabla 4 Distribución de tiempo de Asesoría Reticular Alumnos regulares

Fuente: Elaboración Propia

Por otra parte, los tiempos de los alumnos irregulares se pueden observar en la Tabla 5.

\begin{tabular}{|l|l|}
\hline \multicolumn{2}{|l|}{ Iregular Tercero/Convalidados } \\
\hline Media & 4.684 \\
\hline Desviacion Estandar & 2.334 \\
\hline Distribucioin & Lognormal \\
\hline Iregular Quinto/Convalidados \\
\hline Media & 19.685 \\
\hline Desviacion Estandar & 8.803 \\
\hline Distribucioin & Lognormal \\
\hline Iregular Septimo/Convalidados \\
\hline Media & 23.22 \\
\hline Desviacion Estandar & 10.97 \\
\hline Distribucioin & Lognormal \\
\hline
\end{tabular}

Tabla 5 Distribución de tiempo de Asesoría Reticular Alumnos regulares

Fuente: Elaboración Propia

Utilizando la información anterior, se alimenta el modelo base de simulación para observar su funcionamiento y poder hacer su validación y verificación.

\section{Definición de medidas de desempeño del Sistema}

Las medidas que se desean observar del sistema tiene que ver con el tiempo de espera de los alumnos en el proceso de inscripción. 
Esto debido a que se presentan muchas inconformidades al momento de llevar a cabo esta acción, sobre todos los alumnos regulares mencionan que tardan mucho en el momento de esperar para que se lleve a cabo su asesoría reticular, de igual forma, los docentes comentan que algunos semestres presentan mayor reto al momento de hacer la reinscripción ya que los alumnos irregulares de semestres avanzados tienen mayor número de materias pendientes lo que provoca que se requiera más tiempo al momento de llevar a cabo su asesoría reticular, por lo que los docentes que les toca los semestres más avanzados tienen mayor ocupación en comparación con los docentes que inscriben los semestres menos avanzados. Por último, debido a que el tiempo de inscripción es de 9:00 a 17:00, con un receso de 14:00 a 15:00 horas se desea saber cuántos alumnos quedan pendientes de inscripción después de las 17:00 horas, ya que para esto se requiere que varios docentes y administrativos se queden a laborar más del tiempo programado lo que ocasiona molestias entre el personal.

\section{Verificación del modelo}

Para llevar la verificación del modelo, este fue presentado al personal encargado de llevar a cabo el proceso de inscripción. Se les mostró la forma en como el modelo corría para ir identificando cada una de las partes de dicho proceso. De igual forma se mostró a los docentes encargados del proceso de asignación de la asesoría académica para que ellos indicasen si consideraban que la forma en cómo se comportaba el modelo era semejante a la realidad, quedando todos de acuerdo con el comportamiento del modelo.

\section{Validación del modelo base y obtención de número óptimo de corridas}

Una vez construido el modelo base, se procede a realizar la validación del modelo, por lo que se realizan de manera arbitraria 10 corridas piloto, con la finalidad de evaluar si los datos reales coinciden con los datos del modelo base. Cabe mencionar que se toma en cuenta como medida de desempeño el tiempo de atención de los alumnos por los docentes en el proceso asesoría reticular. Debido a que se tienen diferentes tipos de alumnos, es decir alumnos regulares e irregulares de distintos semestres, se seleccionó aleatoriamente el tipo de alumno y el semestre para llevar a cabo la validación quedando los alumnos de tercer semestre regulares.
Con los resultados de las corridas piloto se hace un análisis con la prueba t-apareada para determinar si los datos obtenidos son válidos. A continuación, se muestran los resultados de la prueba t-apareada, buscando la mínima diferencia entre los valores reales (x) y los valores arrojados por el modelo (y). (Ver tabla 6)

\begin{tabular}{|l|r|r|r|r|}
\hline Corrida & \multicolumn{1}{l}{$\mathbf{X i}$} & \multicolumn{1}{c}{ yi } & \multicolumn{1}{c|}{ zi } & (zi-z10) $^{\mathbf{2}}$ \\
\hline 1 & 2.25 & 2.7 & -0.45 & 4.12 \\
\hline 2 & 2.12 & 1.9 & 0.22 & 1.85 \\
\hline 3 & 3.17 & 3.45 & -0.28 & 3.46 \\
\hline 4 & 1.58 & 1.52 & 0.06 & 2.30 \\
\hline 5 & 2.92 & 0.63 & 2.29 & 0.50 \\
\hline 6 & 4.67 & 1.13 & 3.54 & 3.83 \\
\hline 7 & 2 & 1.25 & 0.75 & 0.69 \\
\hline 8 & 11.4 & 1.78 & 9.62 & 64.59 \\
\hline 9 & 1.7 & 2.70 & -1.00 & 6.65 \\
\hline 10 & 2.02 & 0.97 & 1.05 & 0.28 \\
\hline Suma & & & & \\
\hline Promedio & 3.38 & 1.80 & 1.58 & 88.27 \\
\hline
\end{tabular}

Tabla 6 Resultados de la t-pareada Fuente: Elaboración Propia

\begin{tabular}{|l|r|r|}
\hline VarZ & 0.98 & 2.685010847 \\
\hline Z10 & 4.24 & 0.990336575 \\
\cline { 2 - 3 } Z10 negativo & -1.08 & 2.659064445 \\
\hline
\end{tabular}

Tabla 7 Datos obtenidos de la t-pareada Fuente: Elaboración Propia

Con los datos obtenidos se llega al siguiente intervalo $(-1.08,4.24)$. En los valores obtenidos se observa que las 10 corridas piloto arrojan valores cercanos a los valores reales, por lo que se determina que el modelo es válido, ya que el intervalo de confianza contiene al cero, indicando que no se tiene diferencia significativa entre los resultados del modelo de simulación y los del sistema real.

El siguiente paso es determinar el número óptimo de corridas, para esto de utilizó la prueba $\mathrm{n}^{*} \beta$. Partiendo de los resultados de las 10 corridas, y tomando estos valores como se presentan en la siguiente Tabla 8.

Finalmente, se utiliza la prueba de $n * \beta$ utilizando la Fórmula (1), tomando el tiempo de atención de los alumnos por los docentes en el proceso asesoría reticular de tercer semestre. 


\begin{tabular}{|l|r|}
\hline Corrida & $\begin{array}{c}\text { Tiempo de atención } \\
\text { del alumno regular } \\
\text { 3 er. Semestre }\end{array}$ \\
\hline 1 & 2.70 \\
\hline 2 & 1.90 \\
\hline 3 & 3.45 \\
\hline 4 & 1.52 \\
\hline 5 & 0.63 \\
\hline 6 & 1.13 \\
\hline 7 & 1.25 \\
\hline 8 & 1.78 \\
\hline 9 & 2.70 \\
\hline 10 & 0.97 \\
\hline
\end{tabular}

Tabla 8 Corridas piloto

Fuente: Elaboración Propia

$n *(\beta)=\min \left\{i \geq n: t_{i-1,1-\alpha / 2} \sqrt{\frac{s^{2}(n)}{i}} \leq \beta\right\}$

Se obtuvieron los siguientes resultados:

\begin{tabular}{|l|r|}
\hline$A$ & 0.05 \\
$B$ & 0.50 \\
S $_{n}{ }_{n}$ & 0.803938272 \\
$\mathbf{X}_{n}$ & 1.80 \\
\hline
\end{tabular}

Tabla 9 Parámetros $\mathrm{n} * \mathrm{~B}$

Fuente: Elaboración Propia

\begin{tabular}{|c|r|r|r|r|r|r|}
\hline $\mathbf{i}$ & \multicolumn{2}{|c|}{$\begin{array}{c}\text { Valor t- } \\
\text { apareada }\end{array}$} & \multicolumn{3}{|l|}{$\mathbf{S}^{\mathbf{2}} / \mathbf{i}$} & \multicolumn{2}{|c|}{$\mathbf{n} \boldsymbol{\beta}$} & \multicolumn{2}{|c|}{} \\
\hline 10 & 2.26 & 0.28 & 0.64 & $<=$ & 0.50 & no \\
\hline 11 & 2.23 & 0.27 & 0.60 & $<=$ & 0.50 & no \\
\hline 12 & 2.20 & 0.26 & 0.57 & $<=$ & 0.50 & no \\
\hline 13 & 2.18 & 0.25 & 0.54 & $<=$ & 0.50 & no \\
\hline 14 & 2.16 & 0.24 & 0.52 & $<=$ & 0.50 & no \\
\hline 15 & 2.15 & 0.23 & 0.50 & $<=$ & 0.50 & $\mathrm{Si}$ \\
\hline 16 & 2.13 & 0.22 & 0.48 & $<=$ & 0.50 & $\mathrm{Si}$ \\
\hline 17 & 2.12 & 0.22 & 0.46 & $<=$ & 0.50 & $\mathrm{Si}$ \\
\hline 18 & 2.11 & 0.21 & 0.45 & $<=$ & 0.50 & $\mathrm{Si}$ \\
\hline 19 & 2.10 & 0.21 & 0.43 & $<=$ & 0.50 & $\mathrm{Si}$ \\
\hline 20 & 2.09 & 0.20 & 0.42 & $<=$ & 0.50 & $\mathrm{Si}$ \\
\hline
\end{tabular}

Tabla 10 Datos $\mathrm{n} * \mathrm{~B}$

Fuente: Elaboración Propia

Como se observar en la Tabla 10, a partir de 15 corridas se logra un valor menor a 0.5 que representa el error que se está dispuesto a soportar como diferencia entre la media real de atención de los alumnos regulares de tercer semestre y la media simulada. Una vez determinado el número óptimo de corridas, se procedió a correr el modelo 15 veces durante el horario de 9:00 de la mañana a las 17:00 horas, dejando una hora de descanso de 14:00 a 15:00 horas, para obtener los resultados y poderlos analizar las medidas de desempeño del sistema.

\section{Resultados}

Una vez que el modelo base fue verificado y validado, el siguiente paso es observar en el modelo las medidas de desempeño del sistema ya indicadas. Como ya se mencionó, la mayoría de las quejas vienen de los alumnos regulares quienes indican que tardan mucho esperando a que se lleve a cabo su asesoría reticular, por lo que este tiempo de esta será una medida a observarse.

Por otra parte, se va a evaluar el porcentaje de ocupación de los docentes tal como opera en este momento el proceso de inscripción. Por último, se verá el número de alumnos atendidos en el horario actual y cuantos quedan pendientes a ser atendidos. Después de llevar a cabo las 15 réplicas, se presentan los resultados del modelo base:

\section{Alumnos de tercer semestre regulares}

De la cantidad de alumnos que entran, son 127 alumnos salen completamente del sistema, es decir, que acaban su proceso dentro del tiempo establecido los cuales han concluido su proceso en los horarios que se establecieron. El tiempo que tardan los alumnos en promedio desde que entran por su boleta y salen con su carga académica autorizada es de 77.30 minutos; su tiempo de espera promedio en todo el proceso es de 42.43 minutos, se puede observar un promedio de tiempo de operación de 4.18 minutos, la mayor parte del tiempo de operación es precisamente el proceso de asesoría reticular, ya que, en el resto de las estaciones, el tiempo de espera es realmente poco.

\section{Alumnos de tercer semestre irregulares/convalidados}

Se pueden observar 32 salidas del sistema, sin quedar algún alumno de este tipo. El tiempo que tardan los alumnos en promedio desde que entran por su boleta y salen con su carga académica autorizada es de 77.30 minutos; su tiempo de espera en promedio en cada fase del proceso es de 42.43 minutos. Se puede apreciar que estos alumnos tardan menos tiempo debido a que existe un área especial para alumnos irregulares de cualquier semestre, por lo que los alumnos irregulares pueden ir tanto a la asesoría de tercer semestre como a la asesoría de los irregulares. Esto provoca por lo tanto que se puedan acelerar un poco más y el tiempo de espera sea menor.

SOLIS-JIMENEZ, Miguel Ángel, CALDERÓN-PALOMARES, Luis Antonio, LOPEZ-LEON, Alí y GONZÁLEZ-SOBAL, Martín. Caracterizacion, simulacion y propuesta de mejora del proceso de reinscripcion en el Instituto Tecnologico Superior de Huatusco. Revista de Gestión Universitaria. 2019 
Por último, se puede apreciar que el tiempo de operación efectivamente es mayor que el tiempo de los alumnos regulares y esto se debe a que al momento de llevar a cabo el proceso de asesoría reticular, estos alumnos requieren mayor cantidad de tiempo debido a que se debe acomodar su horario.

\section{Alumnos de quinto semestre regulares}

Hay entre 63 y 64 salidas del sistema; quedando 36 alumnos en la fila, como ya se mencionó en el caso de los alumnos de tercer semestre, los que no son atendidos en el tiempo normal, deberán ser atendidos fuera de este, provocando que tanto docentes como personal de servicios escolares tengan que quedarse horas extras. El tiempo que tardan los alumnos en promedio desde que entran por su boleta y salen con su carga académica autorizada es de 186.11 minutos, lo anterior debido a que alumnos irregulares de este semestre son más difíciles para llevar a cabo su asesoría reticular por parte de los docentes, de manera que el docente que se encuentra encargado de este semestre; su tiempo de espera en promedio en el proceso es de 31.23 , y se puede apreciar un tiempo de bloqueo bastante elevado de 148.74 minutos, esto debido a que alumnos de este semestre y de séptimo son los que siguen llegando por la tarde, de manera que les toca la hora de receso en espera.

\section{Alumnos de quinto semestre irregulares/convalidados}

Con respecto a estos alumnos se puede observar entre 32 y 33 salidas en promedio del sistema; quedando un alumno en la fila. El tiempo que tardan los alumnos en promedio desde que entran por su boleta y salen con su carga académica autorizada es de 161.11 minutos, de igual forma que pasó con los alumnos irregulares de tercer semestre, al haber un docente dedicado a los irregulares, permite que el tiempo de espera de estos en promedio se reduzca considerablemente con relación a los regulares de quinto semestre. Su tiempo de espera en promedio en cada fase del proceso es de 26.23 minutos y se aprecia también un elevado tiempo de bloqueo 110.94 minutos, debido a la situación ya expuesta con los alumnos regulares de quinto semestre.

\section{Alumnos de séptimo semestre regulares}

Hay entre 38 y 37 salidas del sistema en promedio; quedando 57 alumnos en la fila. El tiempo que tardan los alumnos en promedio desde que entran por su boleta y salen con su carga académica autorizada es de 184.49 minutos; su tiempo de espera en promedio en cada fase del proceso es de 30.43 minutos, y un tiempo de bloqueo de 149.36 minutos.

\section{Alumnos de séptimo semestre irregulares/convalidados}

Hay entre 16 y 17 salidas del sistema en promedio; quedando 1 alumno en la fila. El tiempo que tardan los alumnos en promedio desde que entran por su boleta y salen con su carga académica autorizada es de 168.48 minutos; su tiempo de espera en promedio en el proceso es de 27.75 minutos y un tiempo promedio de bloqueo de 114.67 minutos.

De los datos anteriores se puede destacar que el tiempo de operación promedio en cada caso de los irregulares siempre fue mayor, siendo de 6.92, 23.51 y 27.57 minutos para los alumnos de tercero, quinto y séptimo respectivamente de los irregular, contra 4.18, 5.65 y 4.21 minutos de los regulares de los mismos semestres. Esto debe llamar la atención ya que los alumnos irregulares consumen mayor tiempo al momento de llevar a cabo su proceso de asesoría reticular y por lo tanto provocan que el promedio de atención se eleve para todos incluyendo a los alumnos regulares.

También se puede observar que en promedio quedan muchos alumnos para ser atendidos fuera del horario programado que es hasta las 17:00 horas, de manera que se observar que al final del día quedan entre 95 y 96 alumnos de todos los semestres, lo que provoca por una parte que esos alumnos deban inscribirse ya sea en horas extra o en los días siguientes, siendo esto un problema para todos los departamentos involucrados en el proceso de reinscripción.

Por otra parte, se puede apreciar que los porcentajes de utilización de los docentes que llevan a cabo las inscripciones son de $75.23 \%$ para el de tercer semestre; de $95.33 \%$ y $95.55 \%$ para el de quinto y séptimo respectivamente. El docente que lleva a cabo el proceso de inscripción de los irregulares se encuentra en un 93.67\% muy cercano a los de quinto y séptimo semestre.

SOLIS-JIMENEZ, Miguel Ángel, CALDERÓN-PALOMARES, Luis Antonio, LOPEZ-LEON, Alí y GONZÁLEZ-SOBAL, Martín. Caracterizacion, simulacion y propuesta de mejora del proceso de reinscripcion en el Instituto Tecnologico Superior de Huatusco. Revista de Gestión Universitaria. 2019 


\section{Propuestas de mejora}

Una vez analizada la situación actual, se procede a presentar propuestas buscando con estas establecer alguna mejora en las medidas de desempeño presentadas. Se llevaron a cabo varias propuestas de mejora, aunque solo se explica la del tercer Escenario ya que fue esta la que mejor desempeño tuvo.

Escenario 1. Utilizar dos docentes para regulares de todos los semestres y dos para los irregulares.

Escenario 2. Utilizar tres docentes para irregulares de todos los semestres y uno para los regulares.

Escenario 3. Dividir a los alumnos según las clasificaciones (regulares, irregulares) por días.

Esta es la mejor opción como se explica a continuación:

1. Para este escenario se plantea que todos los alumnos regulares se inscriban en un solo día con un horario de cinco horas, es decir, de 9:00 a 14:00 hrs., los cuales van a ser atendidos por los cuatro docentes, y el día posterior vendrán los alumnos irregulares con un horario de 9:00 hrs. A 17:00 hrs., con su respectivo receso, donde los atenderán los cuatro docentes.

2. Al analizar este escenario respecto al original se puede observar una mejoría en las salidas de los alumnos del sistema ya que más del $98 \%$ de los alumnos son atendidos de los diversos semestres. Con base al tiempo que tardan los alumnos dentro del sistema, es considerablemente menor al presentado en el primer modelo, y los tiempos de bloqueo se puede afirmar que son casi nulos, ya que tardan menos de medio minuto, todo ello respecto al modelo original.

3. Respecto a la utilización de los docentes, en el proceso de asignación de cargas, se observa una mejoría en dichos porcentajes, por lo que los docentes se encuentran ocupados por debajo del 50\% del tiempo.
4. Con respecto a los resultados del escenario tres de los alumnos irregulares, se observa que todos los alumnos son atendidos en su totalidad, sin dejar alguno fuera. El tiempo que tardan dentro del sistema se reduce en cada uno de los semestres, al igual que el tiempo de bloqueo es menor, respecto al escenario original.

5. Con relación a la utilización de los docentes, en el proceso de asignación de cargas, se observa en los porcentajes que los docentes se encuentran ocupados por encima del $90 \%$ del tiempo, esto debido a que como son alumnos irregulares necesitan asesorarlos mejor para la realización de la carga académica.

6. Cabe hacer mención en este escenario que no fue necesario cumplir con todo el horario para atender a los alumnos irregular, es decir, a las 14:00 hrs en el modelo de simulación ya se habían atendido al total de los alumnos, esto mejora considerablemente con relación a la situación actual y a los tiempos de los otros escenarios.

\section{Conclusiones}

Escenario 1. Con el análisis de este escenario se puede apreciar que se benefician los alumnos regulares, sin embargo, los alumnos irregulares se están viendo perjudicados ya que el número de alumnos atendidos es menor.

Escenario 2. Con este escenario se puede apreciar que los alumnos de tercer semestre regulares, se ven afectados al ser atendidos, siendo menor su número de salidas, y por consiguiente los bloqueos son mayores, a pesar de no tener problemas para generar su carga académica. Sin embargo, para los alumnos irregulares hasta cierto punto se ven beneficiados, de lo contrario se espera es que se vea reflejado que no se afecten especialmente los alumnos regulares ya que estos hacen lo posible por mantenerse al margen respecto a la acreditación de sus materias 
Escenario 3. Con el análisis observado en la propuesta de mejora tres, la cual indica que se han divididos por días a los alumnos dependiendo la acreditación de sus materias, es decir en regulares e irregulares, un día para regulares, y el posterior para irregulares. Siendo esta opción la de mejor desempeño ya que todos los alumnos serán atendidos dentro del horario establecido ya que en los otros escenarios se observa que a pesar de que son atendidos todos el horario se alarga, sin embargo al correr este escenario se observa que hay tiempo libre para los profesores, es decir se ven menos presionados especialmente para alumnos regulares, aunque para alumnos irregulares se analiza que el porcentaje de utilización de los profesores es mayor a 90\%, se sabe que es a los alumnos que para generar su carga académica es más minucioso el proceso al ir verificando que no choquen horarios en las materias que van a cursar en su próximo semestre.

\section{Referencias}

Coss Bu, Raúl, Simulación un enfoque práctico, Editorial Limusa, México, 1982.

Enetchile, ¿Qué es simulación?, obtenido de la Red Mundial el 5 de septiembre de 2001, http://www.enetchile.com/simulart-

PROMODEL Ver. Estudiantil.htm

Law, Averill M., W.D. Kelton, Simulation Modeling and Analysis, Editorial Mc Graw Hill, Singapure, 2002.

Moras S., Constantino G., Notas del curso de Simulación I, Instituto Tecnológico de Orizaba, agosto-diciembre de 2001.

Moras S., Constantino G., Notas del curso de Simulación II, Instituto Tecnológico de Orizaba, febrero-junio de 2002.

Shannon, Robert E., Simulación de sistemas, Editorial Trillas, México, 1988. 